\section{Incidence, presenting features, and diagnosis of cicatrising conjunctivitis in the United Kingdom}

\author{
Abstract \\ Purpose Cicatrising conjunctival disorders \\ are uncommon, and are difficult to diagnose \\ and manage. This study was designed to \\ assess the annual incidence and underlying \\ diagnosis of patients with cicatrising \\ conjunctivitis (CC) within the United \\ Kingdom. \\ Methods Clinical data of newly diagnosed \\ cases of $\mathrm{CC}$ were reported via the British \\ Ophthalmological Surveillance Unit at \\ diagnosis and at $\mathbf{1 2}$ months follow-up. \\ Results A total of $50(61 \%)$ ocular mucous \\ membrane pemphigoid (OcMMP), 16 (20\%) \\ Stevens-Johnson syndrome/toxic epidermal \\ necrolysis (SJS-TEN) and $16(20 \%)$ other \\ causes of $\mathrm{CC}$, equating to an incidence of 0.8 , \\ 0.2 , and 0.2 per million, respectively, were \\ reported. Although diagnosis of SJS-TEN \\ was usually within a median of 7 days of \\ symptom-onset, that for OcMMP and other \\ CC was a median 225 days for both. At \\ diagnosis, 64/163 (39\%) eyes had moderate/ \\ severe conjunctival inflammation, and \\ 102/164 (62\%) had symblepharon formation. \\ Although 43/82 (52\%) patients were \\ commenced on immunosuppression or had \\ this therapy modified, at follow-up there was \\ an increase in the number of symblepharon, \\ despite control of inflammation $(P<\mathbf{0 . 0 0 1})$. \\ Mortality only occurred in the SJS-TEN \\ group (4/16 (25\%)). \\ Conclusion CC has a substantial morbidity \\ and for non-SJS-TEN causes, diagnosis is \\ frequently delayed. The proportion of \\ patients given immunosuppressive therapy to \\ prevent disease progression may be less than \\ optimal. These data highlight the need for \\ developing patient access to specialist- \\ designated centres with expertise in CC.
}

CF Radford ${ }^{1,4}$, S Rauz ${ }^{2,4}$, GP Williams², VPJ Saw ${ }^{1,3}$ and JKG Dart ${ }^{1,3}$

Eye (2012) 26, 1199-1208; doi:10.1038/eye.2012.119; published online 22 June 2012

Keywords: prospective incidence; surveillance; ocular surface; conjunctival scarring

\section{Introduction}

Cicatrising conjunctivitis (CC) is a rare but sightthreatening group of disorders for which early diagnosis and appropriate treatment are essential. ${ }^{1}$ Conjunctival scarring and chronic inflammation lead to persistent ocular discomfort, limbitis, limbal epithelial stem cell deficiency, and ocular surface failure with subsequent blinding keratopathy. Although the majority of cases have mucous membrane pemphigoid (MMP), a systemic, 'type 2 ' autoimmune disease ${ }^{2}$ with ocular involvement in about $70 \%$ of cases (ocular MMP, OcMMP), ${ }^{3}$ other causes of either slowly progressive or progressive $\mathrm{CC}$ include atopic keratoconjunctivitis (AKC), Sjögren's syndrome (SS), ocular rosacea, Stevens-Johnson Syndrome (SJS) and its more severe form, toxic epidermal necrolysis (TEN), drug-induced conjunctival cicatrisation (DICC), graft $v$ s host disease (GVHD), linear IgA disease, epidermolysis bullosa acquisita (EBA), and mucocutaneous paraneoplastic disorders, some of which can be clinically indistinguishable from OcMMP., ${ }^{1,4}$

Although early diagnosis and treatment are important, ${ }^{1}$ the diseases are rare and most individual clinicians see few cases. This may lead to failure to recognise the clinical features in the early stages of disease and result in delayed or suboptimal treatment.

The incidence of CC is largely unknown. Several European studies have attempted to measure the incidence of the systemic diseases
${ }^{1}$ Moorfields Eye Hospital NHS Foundation Trust, London, UK

${ }^{2}$ Academic Unit of Ophthalmology and Centre for Translational Inflammation Research, School of Immunity and Infection, College of Medical and Dental Sciences, Birmingham and Midland Eye Centre, University of Birmingham, Birmingham, UK

${ }^{3} \mathrm{NIHR}$ Biomedical Research Centre, Moorfields Eye Hospital NHS Foundation Trust and the UCL Institute of Ophthalmology, London, UK

Correspondence:

S Rauz, Academic Unit of Ophthalmology and Centre for Translational Inflammation Research, School of Immunity and Infection, College of Medical and Dental Sciences, Birmingham and Midland Eye Centre, University of Birmingham, Dudley Road, Birmingham B18 7QU, UK

Tel: + 44 (0)121 507 6849;

Fax: + 44 (0)121 5076853

E-mail: s.rauz@bham.ac.uk

${ }^{4}$ These authors contributed equally and share the role of first author.

Received: 2 February 2012 Accepted in revised form: 20 May 2012 Published online: 22 June 2012 
that may cause CC. A prospective study of patients presenting to dermatologists with subepidermal bullous diseases in three French regions showed a 1.13 per million incidence of MMP. ${ }^{5}$ In a separate French study, the incidence of linear IgA disease and EBA were reported as 0.48 and $0.17-0.26$ per million, respectively; ${ }^{5}$ a slightly lower incidence of the same diseases was reported in a German study, ${ }^{6}$ highlighting the likelihood of regional variance, possibly due to genetic and environmental influences. For the SJS-TEN spectrum of disorders, the annualised incidence in Germany has been estimated as 1.89 per million, ${ }^{7}$ with ocular involvement in circa $70 \%$ and $50 \%$ of cases, respectively, ${ }^{8}$ and a mortality of $25 \%$. $^{7}$

There are no epidemiological data regarding conjunctival scarring disorders in UK. Given the chronic nature of CC and the impact these patients have on clinical service provision, frequently requiring lifelong follow-up and resource thirsty management strategies, a national survey was undertaken in collaboration with the British Ophthalmological Surveillance Unit (BOSU) using their methodology. ${ }^{9}$ Our study specifically aimed to establish (i) the incidence of CC within the United Kingdom, (ii) assess patients' clinical features at the time of diagnosis and at 12 months follow-up, and (iii) to map the distribution of patients as a first step in planning of a network of collaborating specialist clinics within the United Kingdom.

\section{Materials and methods}

Cases of CC were collected prospectively through the BOSU, using previously described methodology, ${ }^{9}$ from January 2008 to December 2008. Ophthalmologists were asked to report cases of newly diagnosed CC, defined as conjunctival inflammation associated with scarring (eg, OcMMP, AKC, ocular rosacea, SS, DICC, GVHD, linear IgA Disease, EBA, ocular surface squamous neoplasia (OSSN), or any case of SJS-TEN). Patients with a history of trachoma, an acute infectious membranous conjunctivitis or trauma (chemical, radiation, heat, mechanical, surgical) were excluded. Reporters completed datasheets relating to demographics and clinical findings at initial presentation and after 12 months follow-up (or the closest clinical review date to 12 months).

For causes of CC other than MMP, a combination of the medical history, ocular and systemic clinical signs, histopathology, or immunopathology where available, were used to establish the diagnosis. For purposes of this study, proven OcMMP cases were defined as those having both a positive direct immunofluorescence (DIF) biopsy result (IgG, IgA, C3 deposition along the basement membrane zone) from any site (eye or extra-ocular), in addition to having the characteristic clinical phenotype for ocular disease (a typical conjunctival fibrosis pattern), with or without MMP diagnosed at an extra-ocular site. Presumed OcMMP cases were defined using the same criteria as for proven OcMMP but with negative, unperformed, or missing DIF biopsy results in the absence of other disorders causing progressive CC including DICC, AKC, SS, and SJS-TEN. ${ }^{10}$ Staging of OcMMP was determined by Mondino and Brown, ${ }^{11}$ and Foster systems. ${ }^{12}$

Only patients resident in the United Kingdom with a new diagnosis of CC made within the 12 months surveillance period (2008) were included in this study. Using mid-2008 population estimates for the 12 UK regions (Office of National Statistics, www.statistics. gov.uk), incidence figures were calculated for each disorder causing CC, and an analysis of short-term (12 months) progression and outcomes was performed.

The study was approved by the BOSU Scientific Committee of the Royal College of Ophthalmologists, and the Multicentre Research Ethics Committee, London (07/MREC02/41). The study was conducted according to the Tenets of the Declaration of Helsinki. Where relevant, data were analysed using the $\chi^{2}$ test for association with the Yates continuity correction for small numbers using SPSS version 15 for Windows (Chicago, IL, USA).

\section{Results}

\section{Reports of cases}

A total of 124 reports of CC and/or SJS-TEN from 31 different hospitals were received via BOSU. The BOSU report card return rate for this period was $77 \%$ (BOSU Personal communication, October 2010). Overall 82 of $124(66 \%)$ were eligible cases, diagnosed as OcMMP $(n=50)$, SJS-TEN $(n=16)$, or other diseases causing CC ('Other CC') $(n=16)$. Of the 42/124 'rejected' reported cases, $17(14 \%)$ presented before the study period, clinical data were not received for $13(11 \%), 7(6 \%)$ were duplicate reports, and $5(4 \%)$ were reported in error. Twelve months follow-up data were available for 40/50 $(80 \%)$ of the OcMMP cases, 9/16 (56\%) of the SJS-TEN cases, and 13/16 (81\%) of the Other CC cases group.

\section{Incidence}

The overall minimum incidence of $\mathrm{CC}$ in the United Kingdom was calculated as 1.3 per million UK population, but with geographical variance: 0.1 for north west England to 4.2 for west Midlands (Figure 1). Upon analysing incidence statistics for each CC category, the minimum incidence per million population was calculated as 0.8 OcMMP (0.6 biopsy-proven, 0.2 presumed), 


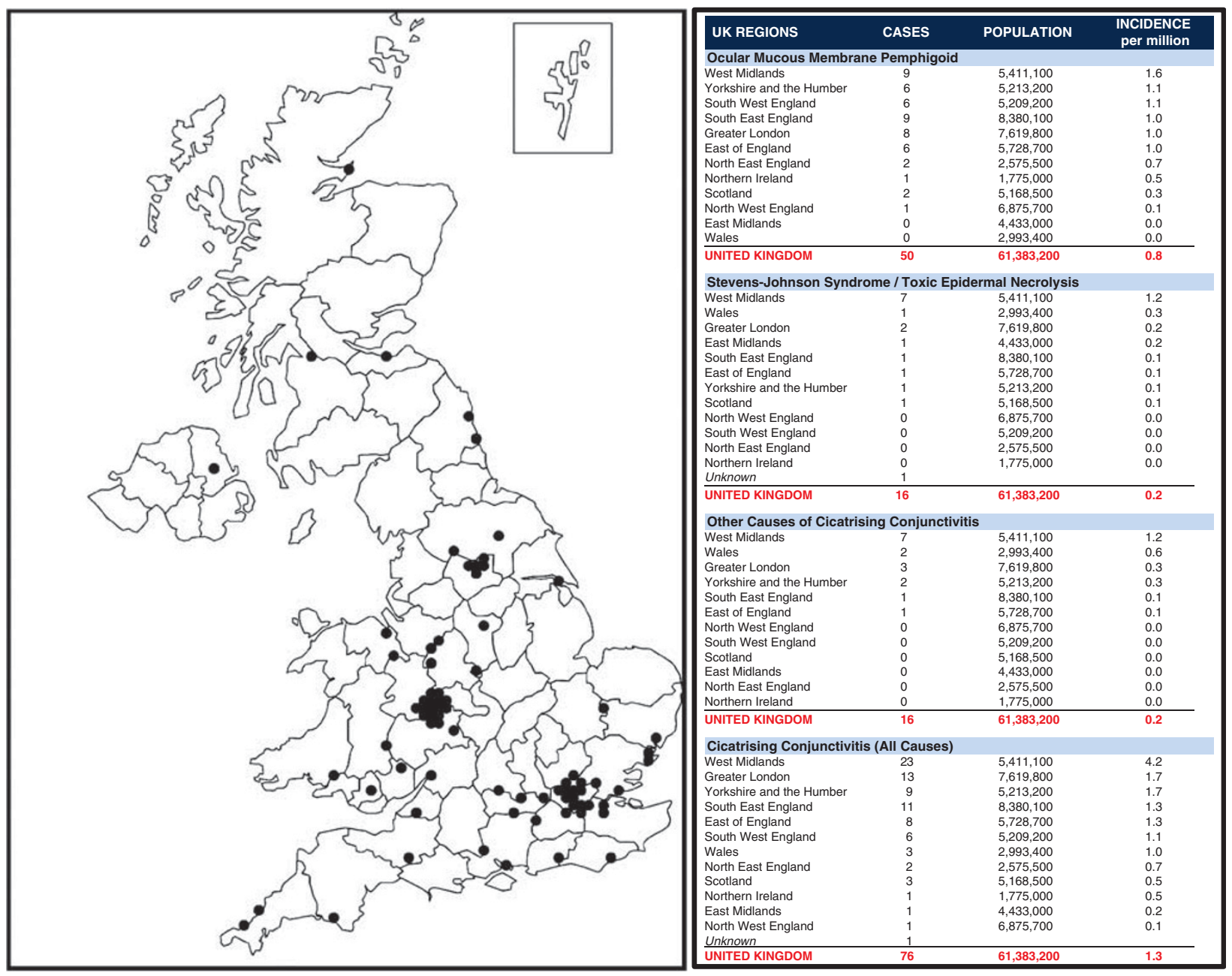

Figure 1 Geographical distribution of newly reported cases of CC in the United Kingdom.

0.2 SJS-TEN, and 0.2 Other CC. A synopsis of the incidence variance according to UK region is provided in Figure 1.

\section{Causes of $\mathrm{CC}$}

Of the 50 cases of OcMMP, almost half (21/50 (42\%)) had not had a tissue biopsy or had biopsy results that were unavailable $(n=4)$; this group included 12 patients with extra-ocular manifestations of the disease. In all, 22 (44\%) were DIF biopsy-positive (conjunctiva, $n=13$; other sites, $n=9)$ and seven (14\%) had a negative biopsy. Indirect immunofluorescence (IIF) studies were positive in 4 of 12 patients, all of whom were DIF biopsy-positive.

Overall, 16 cases of SJS-TEN were reported of whom conjunctival scarring or acute conjunctival adhesion formation was present in 4/5 (80\%) of SJS and 6/11 (55\%) of TEN cases. Concurrent skin manifestations, oral disease, or other mucous membrane involvement were common (SJS, 14/16 (88\%); TEN, 10/16 (63\%)).

Of the remaining 16 cases, $\mathrm{CC}$ had been caused by drugs $(n=3)$, ocular rosacea $(n=3), \operatorname{GVHD}(n=2)$, lichen planus/sclerosis $(n=2), \operatorname{AKC}(n=3), \operatorname{OSSN}(n=1)$, linear IgA disease $(n=1)$, or SS $(n=1)$, with systemic manifestations in $8 / 16(50 \%$; skin $(n=6)$, mouth $(n=2)$, other mucous membranes $(n=2))$.

\section{Diagnostic delay}

Diagnosis of SJS-TEN was significantly quicker (median 7 (range 1-120) days) than for OcMMP or other causes of $\mathrm{CC}$, probably because of its acute presentation vs the more insidious presentations for OcMMP and Other CC $(P=0.01)$. Among patients with OcMMP, absence of preceding extra-ocular manifestations was significantly associated with diagnostic delay $(P=0.019)$ (Table 1$)$. 
Table 1 Conjunctival cicatrisation: summary of incidence, diagnostic delay and patient characteristics

\begin{tabular}{|c|c|c|c|c|}
\hline & \multicolumn{2}{|c|}{$\begin{array}{c}\text { Ocular mucous } \\
\text { membrane pemphigoid } \\
\text { (OcMMP) }\end{array}$} & \multirow{2}{*}{$\begin{array}{c}\text { Stevens-Johnson } \\
\text { syndrome/toxic epidermal } \\
\text { necrolysis (SJS-TEN) }\end{array}$} & \multirow{2}{*}{$\begin{array}{c}\begin{array}{c}\text { Other cicatrising } \\
\text { conjunctivitis } \\
(\text { Other CC })^{\mathrm{a}}\end{array} \\
16\end{array}$} \\
\hline Number of reported cases & 50 & & & \\
\hline $\begin{array}{l}\text { Incidence per million (assuming UK } \\
\text { population of } 61383200 \text { ) }\end{array}$ & 0.8 & & 0.2 & 0.2 \\
\hline \multicolumn{5}{|l|}{ Diagnostic delay } \\
\hline Range & $7-3650$ & & $1-120$ & $16-7300$ \\
\hline Median & 225 & & 7 & 225 \\
\hline \multirow[t]{2}{*}{ Mean $( \pm S D)$} & $605( \pm 897)$ & & $18.6( \pm 31.07)$ & $751( \pm 1779)$ \\
\hline & $\begin{array}{l}\text { With preceding extra- } \\
\text { ocular signs, } n=21^{\mathrm{b}}\end{array}$ & $\begin{array}{l}\text { Without preceding extra- } \\
\text { ocular signs, } n=26^{c}\end{array}$ & & \\
\hline Range & 21-1155 & $7-3650$ & & \\
\hline Median & 180 & 405 & & \\
\hline Mean $( \pm S D)$ & $292^{\mathrm{d}}( \pm 326)$ & $915^{\mathrm{d}}( \pm 1131)$ & & \\
\hline \multicolumn{5}{|l|}{ Patient characteristics } \\
\hline Age, mean/median (range) years & $67.1 / 71(20-90)$ & & $40 / 42(5-100)$ & $60.8 / 67.5(14-89)$ \\
\hline Gender, male: female $(\%)$ & $29: 21(58: 42)$ & & $4: 12(25: 75)$ & $9: 7(56: 44)$ \\
\hline Extra-ocular features, n (\%) & $32(64)$ & & $16(100)$ & $8(50)$ \\
\hline Oral & $28(56)$ & & $14(88)$ & $2(13)$ \\
\hline Skin & $10(20)$ & & $16(100)$ & $6(38)$ \\
\hline Other mucous membranes & $6(12)$ & & $10(63)$ & $2(13)$ \\
\hline Autoimmune disease $(\%)^{\mathrm{e}}$ & $8(16)$ & & 0 & $1(6)$ \\
\hline Ocular comorbidity $(\%)^{\mathrm{f}}$ & $17(34)$ & & 0 & $5(31)$ \\
\hline
\end{tabular}

${ }^{a}$ Other causes of cicatrising conjunctivitis were: drug toxicity (3), ocular rosacea (3), atopic conjunctivitis (3), graft $v$ s host disease (2), lichen planus/lichen sclerosis (2), ocular surface squamous neoplasia (1), linear IgA disease (1), and Sjögren's syndrome (1).

${ }^{b}$ Excluding two with unknown symptom duration.

'Excluding one with unknown symptom duration.

${ }^{\mathrm{d}}$ Absence of preceding extra-ocular signs was significantly associated with diagnostic delay $(t=2.4391, P=0.019)$.

eRheumatoid arthritis (RA) (2), RA and ulcerative colitis (2), RA and pernicious anaemia (2), RA and hypothyroidism (2), hypothyroidism (5), Crohn's disease and Grave's disease (2), Sjögren's syndrome (2). All but one of these patients had OcMMP.

${ }^{\text {f}}$ Cataract (20), glaucoma (9), amblyopia (8), maculopathy (6), retinal detachment (4), thyroid eye disease (3), chronic anterior uveitis (2), diabetic retinopathy (2), metastatic endophthalmitis (2), recurrent herpes simplex virus ulcers (2).

\section{Patient characteristics}

Demographic data are detailed in Table 1. The median age (range) for patients with OcMMP and Other CC was 71 (20-90) and 67.5 (14-89) years, respectively, whereas for SJS-TEN patients was 42 (5-100) years. Interestingly, a slight predominance of male patients in OcMMP or Other CC contrasted with the female predominance in SJS-TEN (12/16 (75\%)). Among patients with OcMMP, $8 / 50(16 \%)$ had other systemic autoimmune disease (eg, rheumatoid arthritis) and about a third had ocular comorbidity (eg, glaucoma, cataract).

\section{Clinical features at diagnosis}

A total of 34 of 150 (23\%) eyes had a best-corrected visual acuity (VA) of $<6 / 18$ at the time of diagnosis. Although symblepharon $(101 / 164(62 \%))$, trichiasis $(57 / 164(35 \%))$, entropion $(37 / 164(23 \%))$, and punctate keratitis $(68 / 164$ $(41 \%))$ were the most common presenting clinical signs
(Table 2) specifically in OcMMP and Other CC eyes, mild/moderate ocular surface inflammation ((29/32) $90 \%$ ) was the principal clinical finding in ocular SJS-TEN. Overall, abnormalities of the tear film or dry eye were found in 106/156 (67.9\%) eyes, whereas limbitis (20/164 $(12 \%))$, persistent epithelial defects $(9 / 164(5 \%))$, keratinisation $(26 / 164(16 \%))$ or corneal involvement (scarring/vascularisation (12/164 (7\%)), microbial keratitis $(22 / 164(13 \%)))$ were less common. OcMMP staging (Table 3) was more advanced when graded by Foster $^{12}$ (stage III/IV 71/100 (71\%)) vs Mondino ${ }^{11}$ (stage III/IV 27/94 (29\%)).

\section{Treatments received before diagnosis}

At the time of diagnosis, immunosuppression was being received by $5 / 50(10 \%)$ of OcMMP patients who had coexisting ancillary autoimmune disease, 9/16 (56\%) of SJS-TEN, and none of the cases of Other CC. The burns intensivists/dermatologists had initiated 
Table 2 Cicatrising conjunctival disorders: prevalence of clinical features at diagnosis and follow up

\begin{tabular}{|c|c|c|c|c|c|c|}
\hline \multirow{2}{*}{$\begin{array}{l}\text { CC category (no. of eyes) } \\
\mathrm{n}(\%)\end{array}$} & \multicolumn{2}{|c|}{ OcMMP (100) } & \multicolumn{2}{|c|}{ SJS-TEN (32) } & \multicolumn{2}{|c|}{ Other CC (32) } \\
\hline & Diagnosis & Follow-up & Diagnosis & Follow-up & Diagnosis & Follow-up \\
\hline \multicolumn{7}{|l|}{ Visual acuity } \\
\hline $6 / 6-6 / 18$ & $80 / 98(82)$ & $57 / 71(80)$ & $13 / 22(59)$ & $10 / 18(71)$ & $23 / 30(77)$ & $21 / 26(81)$ \\
\hline $6 / 24-6 / 36$ & $8 / 98(9)$ & $5 / 71(7)$ & $4 / 22(18)$ & $0 / 18(0)$ & $3 / 30(10)$ & $2 / 26(8)$ \\
\hline $6 / 60-3 / 60$ & $3 / 98(3)$ & 3/71 (4) & $1 / 22(5)$ & $0 / 18(0)$ & $3 / 30(10)$ & $3 / 26(12)$ \\
\hline$<3 / 60$ & $7 / 98(7)$ & 6/71 (9) & $4 / 22(18)$ & $4 / 18(29)$ & $1 / 30(3)$ & $0 / 26(0)$ \\
\hline Trichiasis & $46 / 100(46)$ & $37 / 80(46)$ & $2 / 32(6)$ & $7 / 16(44)$ & $9 / 32(28)$ & $9 / 26(35)$ \\
\hline Entropion & $28 / 100(28)$ & $19 / 80(24)$ & $2 / 32(6)$ & $0 / 16(0)$ & $7 / 32(22)$ & $3 / 26(12)$ \\
\hline \multicolumn{7}{|l|}{ Conjunctival inflammation } \\
\hline Absent & 26/99 (26) & $57 / 80(71)$ & $3 / 32(9)$ & $12 / 16(75)$ & $3 / 32(9)$ & $21 / 26(81)$ \\
\hline Mild & 33/99 (33) & $17 / 80(21)$ & $18 / 32(56)$ & $2 / 16(13)$ & $16 / 32(50)$ & $4 / 26(15)$ \\
\hline Moderate & 25/99 (25) & $6 / 80(8)$ & $11 / 32(34)$ & 2/16 (13) & $11 / 32(34)$ & $0 / 26(0)$ \\
\hline Severe & 15/99 (15) & $0 / 80(0)$ & $0 / 32(0)$ & $0 / 16(0)$ & $2 / 32(6)$ & $1 / 26(4)$ \\
\hline \multicolumn{7}{|l|}{ Lower fornix shrinkage } \\
\hline $0-25 \%$ & 33/94 (35) & $27 / 76(36)$ & $28 / 32(88)$ & $9 / 16(56)$ & $17 / 32(53)$ & $16 / 24(67)$ \\
\hline $25-50 \%$ & $34 / 94(36)$ & $19 / 76(25)$ & $2 / 32(6)$ & $6 / 16(38)$ & $11 / 32(34)$ & $6 / 24(25)$ \\
\hline $50-75 \%$ & 16/94 (17) & $16 / 76(21)$ & $2 / 32(6)$ & $0 / 16(0)$ & $2 / 32(6)$ & $2 / 24(8)$ \\
\hline $75-100 \%$ & $11 / 94(12)$ & $14 / 76(18)$ & $0 / 32(0)$ & $1 / 16(6)$ & $2 / 32(6)$ & $0 / 24(0)$ \\
\hline \multicolumn{7}{|l|}{ Symblepharon } \\
\hline Absent & 14/100 (14) & $10 / 80(13)$ & $20 / 32(63)$ & 2/16 (13) & $9 / 32(28)$ & $6 / 26(23)$ \\
\hline Plica loss only & $16 / 100(16)$ & $10 / 80(13)$ & $0 / 32(0)$ & $4 / 16(25)$ & $4 / 32(13)$ & $1 / 26(4)$ \\
\hline One symblepharon & $26 / 100(26)$ & 15/80 (19) & $8 / 32(25)$ & 2/16 (13) & $16 / 32(50)$ & $4 / 26(15)$ \\
\hline Two or more symblephara & $33 / 100(33)$ & $35 / 80(44)$ & $4 / 32(13)$ & $8 / 16(50)$ & $3 / 32(9)$ & $13 / 26(50)$ \\
\hline Ankyloblepharon & $11 / 100(11)$ & $10 / 80(13)$ & $0 / 32(0)$ & $0 / 16(0)$ & $0 / 32(0)$ & $2 / 26(8)$ \\
\hline \multicolumn{7}{|l|}{ Tear film } \\
\hline Normal & 26/92 (28) & $20 / 76(26)$ & $12 / 32(38)$ & $0 / 16(0)$ & 12/32 (38) & $12 / 24(50)$ \\
\hline Reduced break-up time & 29/92 (32) & $28 / 76(37)$ & $12 / 32(38)$ & $8 / 16(50)$ & $8 / 32(25)$ & $12 / 24(50)$ \\
\hline Dry eye & $37 / 92(40)$ & $28 / 76(37)$ & $8 / 32(25)$ & $8 / 16(50)$ & $12 / 32(38)$ & $0 / 24(0)$ \\
\hline \multicolumn{7}{|l|}{ Ocular surface keratinisation } \\
\hline No keratinisation & $81 / 100(81)$ & $69 / 80(86)$ & $28 / 32(88)$ & $10 / 16(63)$ & 29/32 (91) & $21 / 24(88)$ \\
\hline Partial keratinisation & $18 / 100(18)$ & $9 / 80(11)$ & $2 / 32(6)$ & $4 / 16(25)$ & $3 / 32(9)$ & $3 / 24(12)$ \\
\hline Whole-surface keratinisation & $1 / 100(1)$ & $2 / 80(2)$ & $2 / 32(6)$ & $2 / 16(13)$ & $0 / 32(0)$ & $0 / 24(0)$ \\
\hline \multicolumn{7}{|l|}{ Other ocular surface signs } \\
\hline Punctate keratitis & $43 / 100(43)$ & $30 / 80(38)$ & $8 / 32(25)$ & $5 / 16(31)$ & $17 / 32(53)$ & $10 / 26(38)$ \\
\hline Persistent epithelial defect & 4/100 (4) & $2 / 80(3)$ & $3 / 32(9)$ & $4 / 16(25)$ & $2 / 32(6)$ & $0 / 26(0)$ \\
\hline Limbitis & $12 / 100(12)$ & $3 / 80(4)$ & $2 / 32(6)$ & $1 / 16(6)$ & 6/32 (19) & $3 / 26(12)$ \\
\hline (History of) Microbial keratitis & $12 / 100(12)$ & $3 / 80(4)$ & $5 / 32(16)$ & $1 / 16(6)$ & $5 / 32(16)$ & $0 / 26(0)$ \\
\hline Central opacities/neovascularisation & $9 / 100(9)$ & $7 / 80(9)$ & $2 / 32(6)$ & $4 / 16(25)$ & $1 / 32(3)$ & $3 / 26(12)$ \\
\hline
\end{tabular}

a range of strategies for the treatment of acute or subacute SJS-TEN, including intravenous immunoglobulins $(n=5)$, oral prednisolone $(n=2)$, intravenous methylprednisolone $(n=2)$, cyclosporin $(n=1)$, and infliximab $(n=1)$ before first ophthalmology examination. Surgical intervention for hitherto unrecognised sequelae of conjunctival scarring were most common in the OcMMP group (OcMMP 10/50 $(20 \%)$ vs SJS-TEN (0) vs Other CC (1/16 (6\%)), and this included entropion surgery (5/66 (8\%)), electrolysis $(4 / 66(6 \%))$, and cryotherapy $(2 / 66(3 \%))$.

\section{Treatment given after diagnosis}

Following diagnosis, more than half of the patients $(43 / 82(52 \%))$ required either initiation of immunosuppression or alteration of the current therapy to a more potent regimen (step-up) ${ }^{13}$ (OcMMP 30/50 (60\%), 
Table 3 Ocular mucous membrane pemphigoid: disease stage at diagnosis and at follow-up

\begin{tabular}{lcc}
\hline & $\begin{array}{c}\text { At diagnosis, } \\
\mathrm{n}(\%)\end{array}$ & $\begin{array}{c}\text { At follow-up, } \\
\mathrm{n}(\%)\end{array}$ \\
\hline Eyes & 100 & 80 \\
& & \\
Mondino staging & & \\
Stage I & $33 / 94(35)$ & $27 / 76(36)$ \\
Stage II & $34 / 94(36)$ & $19 / 76(25)$ \\
Stage III & $16 / 94(17)$ & $16 / 76(21)$ \\
Stage IV & $11 / 94(12)$ & $14 / 76(18)$ \\
& & \\
Foster staging & & \\
Stage I & $8 / 100(8)$ & $5 / 78(6)$ \\
Stage II & $21 / 100(21)$ & $11 / 78(14)$ \\
Stage III & $59 / 100(59)$ & $52 / 78(67)$ \\
Stage IV & $12 / 100(12)$ & $10 / 78(13)$ \\
& & \\
Disease progression & & \\
Mondino & & $17 / 66(26)$ \\
$\quad$ Worsened by at least one stage & & $49 / 66(74)$ \\
$\quad$ No change & & 8 \\
$\quad$ Already stage IV & & 6 \\
$\quad$ Could not assess & & \\
Foster & & \\
$\quad$ Worsened by at least one stage & & \\
$\quad$ No change & & \\
$\quad$ Already stage IV & & \\
$\quad$ Could not assess & & \\
\hline
\end{tabular}

Mondino and Brown: (I) $0-25 \%$, (II) $25-50 \%$, (III) $50-75 \%$, (IV) $75-100 \%$ loss of inferior fornix. Foster: (I) subconjunctival scarring and fibrosis, (II) fornix foreshortening of any degree, (III) presence of any degree of symblepharon, (IV) end-stage cicatricial pemphigoid.

SJS-TEN 10/16 (63\%), Other CC 4/16 (25\%)), and the most commonly prescribed agents were glucocorticoids $(23 / 82(28 \%))$, dapsone $(12 / 82(15 \%))$, mycophenolate $(12 / 82(15 \%))$, and cyclophosphamide (9/82 (11\%)). Intravenous immunoglobulin was restricted to the SJS-TEN patients $(7 / 16(44 \%))$. The most commonly prescribed topical medications were steroids $(51 / 82$ $(62 \%))$ and lubricants $((50 / 82(61 \%))$. The distribution of treatment strategies among all three groups is summarised in Table 4.

\section{Patient follow-up}

For 20 patients follow-up was not available owing to patient mortality ( $n=4$, all SJS-TEN), failure of the reporting ophthalmologists to supply follow-up data $(n=6)$, or patients being lost to follow-up $(n=10)$. For the $62 / 82(76 \%)$ remaining patients for whom further data were received, the length of follow-up ranged from 116 to 657 days, with a median of 397 days.

At follow up, 88/115 (77\%) of eyes had a VA of $6 / 18$ or better (Table 2). Although there was significantly less conjunctival inflammation compared with diagnosis $(P<0.001)$ in all groups (Table 2$)$, there was an increase in the number of symblepharon (OcMMP 11/78 (14\%), SJS-TEN 2/16 (13\%), Other CC 7/26 (27\%)), indicating progression of conjunctival cicatrisation. This was supported by a subanalysis of the OcMMP group, where eyes had advanced in stage according to both Mondino- (17/66 (26\%)) and Foster- (11/69 (16\%)) staging systems (Table 3 ).

A total of 30 of $62(48 \%)$ patients remained on immunosuppression at the time of follow-up, of which $9(30 \%)$ had a further 'step-up' of treatment during this period; the majority of whom were in the OcMMP cohort (Table 4). Surgical intervention was required for 15 (24\%) patients and this consisted of entropion surgery (8), lash electrolysis (5), cryotherapy (3), amnion grafting (2), surgical division of symblepharon (1), superficial keratectomy (1), and tectonic corneal transplant (1).

\section{Discussion \\ Incidence}

This is the first study to estimate the incidence of conjunctival scarring disorders in any country. Limitations of the study are the 77\% BOSU card return rate and the 13 reported patients (a potential 14\% loss of incident cases) for whom no data were received. One of the first BOSU studies ${ }^{14}$ identified a similar loss of 9\%, but the UK's National Patient Safety Agency and National Research Ethics Service guidelines continue to prevent the recording of the patient hospital number on the report card, which would overcome this problem. The data presented in this manuscript should therefore be considered an estimate of the minimum incidence.

Despite this limitation, the incidence of OcMMP in the United Kingdom (0.8 per million) appears to be in keeping with those reported in France ${ }^{5}$ and Germany. ${ }^{6}$ A total of $90 \%$ of patients with acute SJS-TEN have ocular involvement and our incidence of 0.2 per million is lower than those of hospitalised cases in Germany, even after allowing for their inclusion of cases without ocular involvement. ${ }^{7}$ This is possibly because many patients in the United Kingdom are examined and managed by dermatologists or Burns Unit personnel according to local guidelines, and only those with ocular involvement resistant to first-line treatments, are referred for specialist ophthalmology opinion. Consistent with other studies, ${ }^{7}$ our mortality rate for SJS-TEN group was $25 \%$. Overall, there was considerable apparent geographical variation in the incidence of cicatrising conjunctival disorders in the United Kingdom, but these data are likely to reflect either differing levels of cooperation among reporting ophthalmologists or lack of awareness of the disease in 
Table 4 Treatment initiated at time of diagnosis and during follow-up

\begin{tabular}{|c|c|c|c|c|c|c|}
\hline \multirow{2}{*}{ CC category (no. of patients) } & \multicolumn{2}{|c|}{$\begin{array}{c}\text { Ocular mucous membrane } \\
\text { pemphigoid }(\mathrm{n}=50)\end{array}$} & \multicolumn{2}{|c|}{$\begin{array}{l}\text { Stevens-Johnson syndrome/ } \\
\text { toxic epidermal necrolysis } \\
\qquad(\mathrm{n}=16)\end{array}$} & \multicolumn{2}{|c|}{$\begin{array}{c}\text { Other cicatrising } \\
\text { conjunctivitis }(\mathrm{n}=16)\end{array}$} \\
\hline & $\begin{array}{l}\text { At diagnosis, } \\
\mathrm{n}=50 \\
\mathrm{n}(\%)\end{array}$ & $\begin{array}{l}\text { At follow-up, } \\
\mathrm{n}=40 \\
\mathrm{n}(\%)\end{array}$ & $\begin{array}{l}\text { At diagnosis, } \\
\mathrm{n}=16 \\
\mathrm{n}(\%)\end{array}$ & $\begin{array}{l}\text { At follow-up, } \\
\mathrm{n}=9 \\
\mathrm{n}(\%)\end{array}$ & $\begin{array}{l}\text { At diagnosis, } \\
\mathrm{n}=16 \\
\mathrm{n}(\%)\end{array}$ & $\begin{array}{l}\text { At follow-up, } \\
\mathrm{n}=13 \\
\mathrm{n}(\%)\end{array}$ \\
\hline \multicolumn{7}{|l|}{ Systemic medication } \\
\hline Any immunosuppressant & $32(64)$ & $26(65)$ & $10(63)$ & $2(22)$ & $4(25)$ & $2(15)$ \\
\hline Corticosteroids & $18(36)$ & $12(30)$ & $4(25)$ & $1(11)$ & $1(6)$ & 0 \\
\hline Dapsone & $10(20)$ & $7(18)$ & 0 & 0 & $2(13)$ & $1(8)$ \\
\hline Mycophenolate & $9(18)$ & $13(33)$ & $2(13)$ & $1(11)$ & $1(6)$ & 0 \\
\hline IV Methyl prednisolone & $3(6)$ & $3(8)$ & $1(6)$ & 0 & 0 & 0 \\
\hline Azathioprine & $6(12)$ & $5(13)$ & 0 & 0 & 0 & 0 \\
\hline Cyclophosphamide & $8(16)$ & $4(10)$ & $1(6)$ & 0 & 0 & $1(8)$ \\
\hline Cyclosporin & $1(2)$ & 0 & $1(6)$ & 0 & $1(6)$ & 0 \\
\hline Methotrexate & $1(2)$ & 0 & 0 & 0 & $1(6)$ & 0 \\
\hline Sulphamethoxypyridazine & $1(2)$ & $1(3)$ & 0 & 0 & 0 & 0 \\
\hline Doxycycline & 0 & 0 & 0 & 0 & $2(13)$ & 0 \\
\hline Other $^{\mathrm{a}}$ & 0 & 0 & $11(69)$ & $2(22)$ & 0 & 0 \\
\hline \multicolumn{7}{|l|}{ Changes in immunosuppression } \\
\hline Stepped up & $30(60)$ & $6(15)$ & $10(63)$ & 0 & $4(25)$ & $1(8)$ \\
\hline Stepped up then down & & $2(5)$ & & 0 & & 0 \\
\hline Stepped down (improvement) & & $4(10)$ & & $3(33)$ & & $1(8)$ \\
\hline $\begin{array}{l}\text { Stepped down (refusal/ } \\
\text { intolerance) }\end{array}$ & & $6(15)$ & & 0 & & $1(8)$ \\
\hline No change & & $15(38)$ & & $2(22)$ & & $1(8)$ \\
\hline Not given immunosuppression & & $7(18)$ & & $3(33)$ & & $9(69)$ \\
\hline \multicolumn{7}{|l|}{ Topical medication } \\
\hline Steroids & $26(52)$ & $22(55)$ & $15(94)$ & $6(67)$ & $10(63)$ & $6(46)$ \\
\hline Lubricants & $31^{\mathrm{b}}(62)$ & $33^{\mathrm{b}}(83)$ & $15(94)$ & $8(89)$ & $15(94)$ & $11(85)$ \\
\hline Other & $9(18)$ & $5(13)$ & $8(50)$ & $6(67)$ & $5(31)$ & $3(23)$ \\
\hline \multicolumn{7}{|l|}{ Surgery during follow-up } \\
\hline Any surgery & & $12(30)$ & & $2(22)$ & & $1(8)$ \\
\hline Entropion surgery & & $8(20)$ & & 0 & & 0 \\
\hline Electrolysis & & $5(13)$ & & 0 & & 0 \\
\hline Cryotherapy & & $2(5)$ & & 0 & & $1(8)$ \\
\hline Amniotic membrane graft & & $1(3)$ & & $1(11)$ & & 0 \\
\hline Division of symblephara & & $1(3)$ & & 0 & & 0 \\
\hline Superficial keratectomy & & $1(3)$ & & 0 & & 0 \\
\hline Tectonic graft & & 0 & & $1(11)$ & & 0 \\
\hline
\end{tabular}

${ }^{a}$ Other systemic medications, for SJS/TEN: IV immunoglobulins (7), ganciclovir (1), infliximab (1), meropenem (1), and vancomicin (1) at diagnosis; IV immunoglobulins (1) and infliximab (1) at the time of follow-up.

${ }^{\mathrm{b}}$ Among OcMMP patients there was an increased use of lubricants since diagnosis, $P=0.008$.

'Other topical medications: antibiotics (15), glaucoma therapy (2), autologous serum (1), cyclosporin (2), mitomicin (1), and sodium cromoglycate (1) at diagnosis; antibiotics (5), glaucoma therapy (3), autologous serum (1), cyclosporin (2), mitomicin (1), retinoic acid (1), and sodium cromoglycate (1) at the time of follow-up.

the areas reporting low incidences, and possibly genetic susceptibility due to differences in ethnic mix, rather than environmental/geographical influences.

\section{Diagnosis}

Diagnostic delay was unusual for SJS-TEN patients, who present with generally more acute disease. Considerable diagnostic delay was common for the other, rarer causes of CC together with those with OcMMP. Delays were particularly marked in patients with no pre-existing or coexisting extra-ocular manifestations, highlighting the importance of considering OcMMP as a differential diagnosis in any case of trichiasis or recurrent conjunctivitis, and the need for performing an early confirmatory conjunctival biopsy (with or without an 
oral mucosal biopsy). It is possible that reluctance to perform a conjunctival biopsy was due to misplaced concern about causing more damage before institution of immunosuppression. We believe that if OcMMP is suspected, biopsy of the conjunctiva, and tissue from other sites of potential involvement, should be part of an acceptable standard of care ${ }^{1}$ and with appropriate precautions, bulbar conjunctival biopsy is safe. ${ }^{15}$ If facilities do not exist for appropriate processing of biopsy material, these patients should be referred to specialist centres for diagnostic work-up. ${ }^{1}$ A range of immunopathological (DIF, IIF) or histopathological methods, may also support the diagnosis of other CC disorders (Linear IgA disease, EBA, SS, AKC, OSSN). Nonetheless, a critical caveat for the diagnosis of immunobullous disease is the sensitivity and specificity of currently available immunofluorescence techniques. For OcMMP, a positive DIF result is found in $60-80 \%$ of patients. ${ }^{1,16,17}$ The diagnosis of MMP in patients with the typical phenotype of OcMMP, but with negative DIF, is a current clinical issue; it has been suggested that biopsynegative patients should not be diagnosed as having MMP. ${ }^{2}$ This recommendation leaves patients, who have the typical phenotype and disease progression of OcMMP, outside any current disease category resulting in delayed diagnosis and treatment. We suggest that rather than categorising biopsy-negative patients as having some other unspecified disease, we recommend that these patients should be accepted as presumed $\mathrm{MMP}^{18}$ while efforts are made to improve the sensitivity of existing diagnostic technologies, and to develop novel alternative tools for diagnosis with improved positive predictive values. Our proposed revision of the immunopathological criteria for the diagnosis of OcMMP are detailed in Table 5.

\section{Management}

A total of 32 of the 50 patients $(64 \%)$ were given immunosuppressive therapy after diagnosis, and $65 \%$ continued to be on immunosuppression at follow up. It is difficult to establish a figure for the proportion of OcMMP patients who require immunosuppression as the publications that describe large cohorts of patients have focused on the use of immunosuppressive therapies for the disease as a whole. ${ }^{19,20}$ However, in a series of 66 patients with a minimum of 2 years follow-up reported in $1996,{ }^{21} 17 / 66$ (26\%) did not have inflammatory disease considered severe enough to justify the risks of immunosuppression, although this might not be the case in a more contemporary case series.

The low proportions of patients in our current paper treated with immunosuppressive therapy might represent a reluctance to use this form of treatment outside specialist centres. At follow-up, all groups were shown to have a significant decrease in the frequency of conjunctival inflammation, but even with immunosuppression, fornix shrinkage progressed in 17/66 (26\%) of eyes with OcMMP. Although prognosis is improved with systemic immunomodulation, ${ }^{12,19}$

Table 5 Proposed revised immunopathological criteria for the diagnosis of ocular mucous membrane pemphigoid

$\begin{array}{ll}\text { Consensus criteria }^{\text {a }} & \text { 1. Direct immunofluorescence - presence of basement membrane zone (BMZ) deposits of IgG, IgA, IgM, } \\ \text { and /or C3. } \\ \text { - Detection of one or combination of the above linear epithelial BMZ immune deposits establishes the diagnosis } \\ \text { of autoimmune MMP. } \\ \text { - Patients with clinical manifestations similar or identical to MMP but in whom epithelial BMZ immune } \\ \text { deposits have not been demonstrated, these patients may be drug-induced or the pathogenesis of the disease } \\ \text { needs to be further elucidated. } \\ \text { 2. Indirect immunofluorescence - presence of IgG and IgA autoantibodies binding to skin BMZ on salt split } \\ \text { epithelial substrate. } \\ \text { - Not all patients with MMP have detectable circulating autoantibodies to the basement membrane zone. } \\ \text { The consensus does not consider these findings to be an absolute criterion. } \\ \text { Proposed revised } \\ \text { Priteria } \\ \text { of ocular mucous membrane pemphigoid PLUS either one of the following: } \\ \text { 1. Positive conjunctival direct immunofluorescence or positive direct immunofluorescence from any other site } \\ \text { (eg, oral mucosa, skin) that meet the current consensus criteria. } \\ \text { Or } \\ \text { 2. Negative direct immunofluorescence from any site and positive indirect immunofluorescence are diagnosed } \\ \text { as having MMP. } \\ \text { Or } \\ \text { 3. Negative immunofluorescence studies (direct or indirect) only when other diseases that may cause this } \\ \text { phenotype have been excluded, are diagnosed as presumed OcMMP. }\end{array}$

${ }^{\mathrm{a}}$ Chan et al. ${ }^{2}$ 
it is important to note that up to $42 \%$ of patients continue to demonstrate progressive conjunctival scarring without clinically manifest inflammation. ${ }^{1,19}$ In addition, $24 \%$ of cases required oculoplastic surgery to minimise secondary ocular surface inflammation induced by deformed lids and misdirected lashes.

\section{Summary}

The overall minimum incidence of CC in the United Kingdom is estimated as 1.3 per million. The proportion of patients with CC having appropriate investigations to confirm the disease is low, and the proportion of OcMMP having immunosuppressive therapy is probably less than optimal to prevent progression of disease, which should be monitored objectively, with clearly defined and validated activity and damage indices of disease. ${ }^{1,22}$ Our data support the need for both specialist oculoplastic and immunosuppression treatment expertise, to appropriately manage patients in the United Kingdom with CC. ${ }^{1}$ Currently, most patients reside in the West Midlands, Yorkshire, London, and the South East of the United Kingdom where there is existing service provision for patients with CC. Improved awareness within areas reporting a low incidence of the disease, and better access to designated specialist centres, can be expected to enable delivery of the expert care that patients with these diseases require if they are to receive optimal diagnostic work-up and management.

\section{Summary}

What was known before

- Cicatrising conjunctivitis (CC) is a rare but sightthreatening group of disorders for which early diagnosis and appropriate treatment are essential.

- Most clinicians see few cases.

- There are no incidence data for conjunctival scarring disorders in the United Kingdom.

What this study adds

- The overall minimum incidence of CC in the United Kingdom is 1.3 per million with geographical variance. There is a wide range in duration of ocular symptoms (7-3650 days) before these diseases are recognised.

- The use of appropriate investigations to confirm the disease diagnosis is low. The proportion of patients receiving optimal therapy to prevent progression of disease is less than ideal.

- Objective assessment instruments with clearly defined and validated activity and damage indices of disease are required. Patients should be reviewed in dedicated specialist centres with expertise in the diagnosis and management of CC.

\section{Conflict of interest}

The authors declare no conflict of interest.

\section{Acknowledgements}

This study was funded by Wellcome Trust, UK (GPW, SR); Moorfields Eye Hospital Unrestricted Grant (JKGD, CR).

\section{Author contributions}

CR, SR, and JD contributed to study design. CR and GPW validated questionnaires. CR and SR lead governance issues relating to the study (MREC application, BOSU submission and approval process). CR collated data. CR and GPW carried out initial data analyses. All authors contributed to revision of data presentation and analysis. CR and SR wrote the first draft of the manuscript and made revisions to subsequent drafts. All authors commented on revision of manuscript.

\section{Disclaimer}

S Rauz has the right to grant on behalf of all authors and does grant on behalf of all authors, an exclusive licence (or non-exclusive for government employees) on a worldwide basis to the Nature Publishing Group, and its Licensees to permit this article (if accepted) to be published in the Eye and any other Nature products and to exploit all subsidiary rights, as set out in the Nature licence.

\section{References}

1 Williams GP, Radford C, Nightingale P, Dart JKG, Rauz S. Evaluation of early and late presentation of patients with ocular mucous membrane pemphigoid to two major tertiary referral hospitals in the United Kingdom. Eye 2011; 25(9): 1207-1218.

2 Chan LS, Ahmed AR, Anhalt GJ, Bernauer W, Cooper KD, Elder MJ et al. The first international consensus of mucous membrane pemphigoid - Definition, diagnostic criteria, pathogenic factors, medical treatment, and prognostic indicators. Arch Dermatol 2002; 138: 370-379.

3 Bernauer W, Itin PH, Kirtschig G. Cicatricial pemphigoid. In: Bernauer W, Dart JKG, Elder MJ (eds). Cicatrising Conjunctivitis. Developments in Ophthalmology Vol. 28 Karger: Basel, 1997, pp 46-63.

4 Bernauer W, Elder MJ, Dart JKG. Introduction to cicatrising conjunctivitis. In: Bernauer W, Dart JKG, Elder MJ (eds). Cicatrising Conjunctivitis. Developments in Ophthalmology Vol. 28, Karger: Basel, 1997, pp 1-10.

5 Bernard P, Vaillant L, Labeille B, Bedane C, Arbeille B, Denoeux JP et al. Incidence and distribution of subepidermal autoimmune bullous skin diseases in three French regions. Arch Dermatol 1995; 131(1): 48-52. 
6 Zillikens D, Wever S, Roth A, Weidenthaler-Barth B, Hashimoto T, Brocker E-B. Incidence of autoimmune subepidermal blistering dermatoses in a region of central Germany. Arch Dermatol 1995; 131(8): 957-958.

7 Rzany B, Mockenhaupt M, Baur S, Schröder W, Stocker U, Mueller J et al. Epidemiology of erythema exsudativum multiforme majus, Stevens-Johnson syndrome, and toxic epidermal necrolysis in Germany (1990-1992): Structure and results of a population-based registry. J Clin Epidemiol 1996; 49(7): 769-773.

8 Power WJ, Ghoraishi M, Merayo-Lloves J, Neves RA, Foster CS. Analysis of the acute ophthalmic manifestations of the erythema multiforme/Stevens-Johnson syndrome/toxic epidermal necrolysis disease spectrum. Ophthalmology 1995; 102(11): 1669-1676.

9 Foot B, Stanford M, Rahi J, Thompson J. The British Ophthalmological Surveillance Unit: an evaluation of the first 3 years. Eye 2003; 17(1): 9-15.

10 Joseph T. Ocular cicatricial pemphigoid. Ophthalmology 2008; 115(9): 1639-1640.

11 Mondino BJ, Brown SI. Ocular cicatricial pemphigoid. Ophthalmology 1981; 88: 95-100.

12 Foster CS. Cicatricial pemphigoid. Trans Am Ophthalmol Soc 1986; 84: 527-659.

13 Rauz S, Maddison PG, Dart JKG. Evaluation of mucous membrane pemphigoid with ocular involvement in young patients. Ophthalmology 2005; 112(7): 1268-1274

14 Radford CF, Minassian DC, Dart JKG. Acanthamoeba keratitis in England and Wales: incidence, outcome, and risk factors. Br J Ophthalmol 2002; 86(5): 536-542.

15 Frith PA, Venning VA, Wojnarowska F, Millard PR, Bron AJ. Conjunctival involvement in cicatricial and bullous pemphigoid: a clinical and immunopathological study. Br J Ophthalmol 1989; 73(1): 52-56.

16 Bernauer W, Elder MJ, Leonard J, Wright P, Dart JKG. The value of biopsies in the evaluation of chronic progressive conjunctival cicatrisation. Graefes Arch Clin Exp Ophthalmol 1994; 232: 533-537.

17 Thorne JE, Anhalt GJ, Jabs DA. Mucous membrane pemphigoid and pseudopemphigoid. Ophthalmology 2004; 111(1): 45-52.

18 Saw VPJ, Dart JKG. Ocular cicatricial pemphigoid - Author Reply. Ophthalmology 2008; 115(9): 1640-1641.

19 Saw VPJ, Dart JKG, Rauz S, Ramsay A, Bunce C, Xing W et al. Immunosuppressive therapy for ocular mucous membrane pemphigoid: strategies and outcomes. Ophthalmology 2008; 115(2): 253-261.

20 Thorne JE, Woreta FA, Jabs DA, Anhalt GJ. Treatment of ocular mucous membrane pemphigoid with immunosuppressive drug therapy. Ophthalmology 2008; 115(12): 2146-2152; e2141.

21 Elder MJ, Bernauer W, Leonard J, Dart JKG. Progression of disease in ocular cicatricial pemphigoid. $\mathrm{Br} J$ Ophthalmol 1996; 80: 292-296.

22 Williams GP, Saw VPJ, Saeed T, Evans ST, Cottrell P, Curnow SJ et al. Validation of a fornix depth measurer: a putative tool for the assessment of progressive cicatrising conjunctivitis. Br J Ophthalmol 2011; 95(6): 842-847.

This work is licensed under the Creative Commons Attribution-NonCommercial-No Derivative Works 3.0 Unported License. To view a copy of this license, visit http://creativecommons.org/licenses/ by-nc-nd/3.0/ 